\title{
EVOLUCIÓN NORMATIVA DEL ANTICIPO DEL IMPUESTO A LA RENTA EN ECUADOR.
}

\author{
REGULATORY EVOLUTION OF THE INCOME TAX \\ ADVANCE IN ECUADOR \\ Néstor Daniel Gutiérrez Jaram ILO* \\ Ena Maritza Feijoó González** \\ Milca NaAra Ore Lana Uшoa*** \\ Docentes de la Universidad Técnica de Machala \\ Universidad Nacional Mayor de San Marcos-UNMSM / Lima-Perú \\ [Recepción: Agosto 2016/ Conformidad: Setiembre 2016]
}

\section{RESUMEN}

El presente trabajo es un análisis detenido y detallado de los aspectos más relevantes, relacionados a la evolución normativa del Anticipo de Impuesto a la Renta, para contribuyentes obligados a llevar contabilidad desde el año 2008 hasta la presente fecha. Se valoran aspectos configurativos tales como, condiciones de cálculo, formas de pago, derecho a devolución y a la exoneración o reducción de su pago. El motivo del estudio es porque que este rubro sigue siendo en la actualidad, un elemento de discusión sobre la pertinencia de su aplicación, ya que se le sigue considerando como confiscatorio e ilegitimo.

Planteando como objetivo realizar un análisis normativo de la evolución en la forma de cálculo, pago y beneficios generales en el Anticipo desde el año 2008 hasta octubre del 2016. Aplicándose la metodología de análisis documental basadas en la normativa legal tributaria vigente, tomando como elemento principal la Ley de Régimen Tributario Interno y su Reglamento.

De igual forma, se toma como base teórica la sentencia de la Corte Constitucional donde se declara la legalidad del Anticipo, así como también a otras investigaciones relacionadas al tema.

Logrando como resultado que desde el año 2016, el Anticipo tome la concepción de Impuesto Mínimo a la Renta, sin derecho a devolución o compensación posterior. $Y$ en el 2016 se da la posibilidad legal para la devolución de una parte del valor cancelado.

\section{Palabras Clave:}

Anticipo, Impuesto a la Renta, base legal, devolución, beneficios.

\begin{abstract}
The present work is detailed analysis of the most relevant aspects related to the normative evolution of the Income Tax Advance for taxpayers who are obliged to keep accounts from 2008 to the present date. Configuration aspects such as calculation conditions, forms of payment, right to return and exemption or reduction of payment are valued. The reason for the study is because the relevance of its application is still, at present, an element of discussion, since it is still considered as confiscatory and illegitimate.

The objective was to carry out a normative analysis of the evolution in the form of calculation, payment and general benefits in the Advance from the year 2008 to October of 2016. Applying the methodology of documentary analysis based on the current legal tax legislation we took as its main element The Internal Tax Regime Law and its Regulations.

Likewise, the sentence of the Constitutional Court that declares the legality of the Anticipation, as well as other investigations related to the subject, are taken as the theoretical basis.

As a result, since 2016, the Advance takes the approach of Minimum Income Tax, with no right to refund or later compensation. And in 2016 the legal possibility for the return of a part of the canceled value is given.
\end{abstract}

\section{Keywords:}

Advance, Income Tax, legal basis, return, benefits.

\footnotetext{
* Doctor en Ciencias Contables y Empresariales - UNMSM. Email: jminano@unmsm.edu.pe

** Doctora en Contabilidad y Ciencias Empresariales - UNMSM. Email. efeijoo@utmachala.edu.ec

*** Magister en Contabilidad y Auditoría - UNMSM. Email. morellana@utmachala.edu.ec
} 


\section{INTRODUCCIÓN}

El Ecuador ha sido un país cuyo sistema tributario, en atención al cumplimiento del principio de suficiencia recaudatoria, ha tenido una estructura histórica de recaudación, donde han prevalecido los impuestos indirectos por sobre los directos. Conla llegada del gobierno del presidente RafaelCorrea Delgado en el año 2007, se promovió a promulgación de unanueva Constitución enlanación,locual tuvo su desenlace el 20 de octubre de 2008, cuando en el Registro Oficial 449 en dicha fecha se publicó la Constitución de la República del Ecuador, y es en esta carta magna donde se determinaron las concepciones básicas del sistema tributario Ecuatoriano. En su artículo 300, se definieron los principios tributarios entre los cuales tenemos el de progresividad, equidad, transparencia y suficiencia recaudatoria, a la vez se establece como prioridad la recaudación de impuestos directos y progresivos. El 29 de diciembre de 2007, en el tercer suplemento del registro oficial 242, se publicó la Ley Reformatoria Para La Equidad Tributaria en El Ecuador, en la cual se dio un hito interesante en el sistema tributario del país, con la creación de una nueva forma de cálculo para liquidar el Impuesto a la Renta de los obligados a llevar contabilidad, basándose en un nuevo concepto de cálculo del Anticipo de Impuesto a la Renta. Lo novedoso del esquema, radicó en que la nueva forma de cálculo de este rubro, responde a un concepto doctrinario de Impuesto mínimo o presuntivo, denominados heterodoxos, de ahí que en el común denominador del colectivo contable y tributario en el país se lo conozca como "Anticipo Mínimo de Impuesto a la Renta”, hecho que es validado, cuando en la propia norma legal tributaria LORTI se menciona el término anticipo mínimo. Desde su promulgación, en el año 2008, se han dado algunas reformas sobre su forma de liquidación, cálculo y beneficios tributarios en relación al Anticipo, siendo la más concluyente y trascendental, que le da la forma de impuesto mínimo, la reforma del año 2010. Al ser una carga impositiva heterodoxa se ha convertido en un tema de mucha discusión e incluso de fuertes controversias legales, por lo que se hace imprescindible detallar un análisis detenido de cómo ha evolucionado en el ámbito legal esta carga fiscal

\section{OBJETIVO}

Realizar un análisis normativo de la evolución en la forma de cálculo, pago y beneficios generales en el Anticipo de Impuesto a la Renta, desde el año 2008 hasta octubre del 2016.

\section{METODOLOGÍA}

El presente trabajo corresponde a un diseño de investigación deductivo-descriptivo, utilizando como herramientas metodológicas, el análisis documental y estadístico, para analizar los efectos en la recaudación del impuesto a la renta, en los diferentes periodos a consecuencia de los cambios normativos en los últimos años. Adoptando como postura el enfoque epistemológico vivencial, para agrupar y detallar los aspectos más relevantes, que le dan forma al Anticipo del Impuesto a la Renta, estos fueron: La forma de cálculo, pago, beneficios generales de devoluciones y de exoneración o reducción de su pago.

\section{BASES TEÓRICAS}

Sin lugar a discusión, los tributos constituyen una de las principales fuentes de financiamiento del presupuesto general del estado en el Ecuador, solventando el gasto público y así procurar promover el desarrollo económico y social de la sociedad. (Masbernat, 2014)

Domínguez y Checa González (2013), ratifican, la clasificación universal común de los tributos en impuestos, tasas y contribuciones especiales o de mejora, haciendo énfasis en que de estos, los impuestos constituyen una de las principales fuentes para generar recaudación, detallando su clasificación en Directos e Indirectos.

La importancia del estudio del Anticipo de Impuesto a la Renta, guarda plena concordancia con el mandato constitucional, que establece que se debe priorizar la recaudación de Impuestos directos y progresivos, recordando que los impuestos directos, son aquellos que gravan directamente al patrimonio o al ingreso, logrando de esta ma- 
nera, la obtención de recursos para el estado de manera justa y equitativa. (Pinto Bernal, 2012)

Por lo tanto, el Anticipo de Renta, al formar parte del propio Impuesto a la Renta se considera como una carga fiscal directa.

En este sentido, la presente investigación tiene como base teórica primaria, la Ley Orgánica de Régimen Tributario Interno, vigente en cada uno de los periodos de tiempo estudiados, esto es desde el año 2008 hasta octubre de 2016, y dentro de este cuerpo normativo existe un énfasis primordial en abordar lo dispuesto por el artículo 41 que, regula todo lo relacionado al Anticipo de Impuesto a la Renta.

Desde el año 2008 hasta la actualidad, el artículo 41 de la LORTI, ha sido objeto de constantes modificaciones a través de leyes especiales $u$ orgánicas, que se han ido publicando en el registro oficial, por tal motivo, se ha centrado mucho el ámbito de vigencia de los elementos normativos relacionados al anticipo en relación a la forma de cálculo, pago, devolución, exoneració y reducciones.

Otro documento de trascendental importancia en el estudio, es la sentencia 006-13-SINCC de fecha 25 de abril de 2013, en la que se declara implícitamente, la legalidad del anticipo y se deriva que el mismo cumple plenamente con los principios constitucionales consagrados en la artículo 300 de la Carta Magna.

Castro, Aguiar \& Sáenz (2013) desarrollaron una investigación, donde se analizó las reformas tributarias en el Ecuador durante el periodo 2001 al 2012, periodo en el cual, ya entró en vigencia la normativa legal que incorporaba en la LORTI al Anticipo Mínimo de Impuesto a la Renta, los resultados fueron que efectivamente las reformas efectuadas, contribuyeron a mejorar la progresividad y a la generación oportuna de ingresos para la caja fiscal.

Por otra parte, Carbajo (2013) también desarrolló un análisis interesante, sobre los modelos tributarios del Impuesto a la Renta, aplicados en toda Iberoamerica,llegando a establecer como resultado, que debido a la incorrecta aplicación y control de las cargas impositivas ortodoxas, se ha llegado a establecer gravámenes "heterodoxos", como en el caso Ecuatoriano conel Anticipo Mínimo del Impuestoala Renta.

\section{RESULTADOS}

Es de trascendental importancia, partir de la aclaración, que aunque el anticipo de Impuesto a la Renta pudiera ser considerado como injusto, confiscatorio, ilegal y cualquier otra connotación que se le quiera dar, dicho tema ya fue discutido y sentenciado por el máximo órgano de justicia del país, La Corte Constitucional.

Gutiérrez (2015) manifiesta, que fueron 13 empresas las que presentaron la acción constitucional, pidiendo se declare la inconstitucionalidad del Anticipo Mínimo de Impuesto a la Renta, entre las principales causales invocadas, se encontraron las afectaciones de orden económico, político, social y legal, que violenta el sistema tributario, al incumplir con los principios tributarios declarados en la Constitución de la República del Ecuador, tales como legalidad, no confiscatoriedad, igualdad y la equidad, debido a que el anticipo no considera la base imponible del propio ejercicio fiscal, también se alegó el incumplimiento de los principios de progresividad y proporcionalidad, ya que en el caso del anticipo la carga impositiva no es progresiva en relación con la riqueza de las personas.

La discusión planteada en líneas anteriores, tuvo su desenlace con la Sentencia No. 006-13-SIN-CC del año 2013, donde se declaró al Anticipo Mínimo del Impuesto a la Renta, como constitucional y por tantosenegarontodaslasaccioneslegalesplanteadas.

Con relación a los principios de equidad e igualdad, la Corte Constitucional determinó, que al existir formulas diferentes de cálculo para cada uno de los grupos de contribuyentes, no se violenta los principios, ya que la diferenciación en el cálculo del anticipo entre obligados y no obligados a llevar contabilidad, es una medida adecuada impuesta por el legislador, tomando como base la capacidad contributiva de los contribuyentes, y que finalmente se cumple uno de los postulados del 
principio de igualdad, que establece la igualdad de trato entre iguales, y desigual trato a los desiguales.

Es de relevancia, destacar que la recaudación que se obtenga de los tributos, siempre debe procurar que se realice con relación a los ingresos que generen los contribuyentes, tratando de que las reformas legales que realicen, consideren la igualdad como principio rector para su aplicación, (Casares $y$ otros, 2015). Por tal motivo, la sentencia incurrida invita a poner mucha atención a la prevalencia de la consideración de la igualdad entre iguales.

En lo que tiene que ver con los principios de progresividad y proporcionalidad, la Corte Constitucional, aclaró que no se trata de un nuevo tributo creado, sino por el contrario, es simplemente un mecanismo de recaudación anticipada del propio Impuesto a la Renta y al final del ejercicio este valor del anticipo pagado, será descontado del pago del propio Impuesto a la Renta, por lo que no existiría el rompimiento de estos dos principios.

Es de considerar, que es este principio el que establece, que los impuestos establecidos se gravarán en función del ingreso y por tanto de la capacidad contributiva de los contribuyentes. (Rodríguez y Vargas, 2015)

Por otra parte y en consideración al principio de legalidad, la instancia judicial definió, que no existe vulneracióndelprincipioyaquelaconcepcióndel Anticipo de Impuesto a la Renta fue establecido por Ley.

Finalmente, en el punto más controversial de la discusión, el incumplimiento del mandato legal de no confiscatoriedad, la Corte Constitucional realizó el análisis, enfocada en el hecho de que el Anticipo de Impuesto a la Renta tiene índoles económicos de importancia, así como fines de índole social, todos relacionados directamente con la política fiscal, ya que se pretende buscar en el contribuyente conductas responsables, por otra parte, en ningún momento se ha coartado el derecho del contribuyente, para que presente bajo específicas circunstancias trámites administrativos, solicitando la devolución del anticipo cada trienio por motivos de caso fortuito o fuerza mayor, o en su defecto solicitar la reducción, exoneración o exención del tributo, lo que da fortaleza al hecho de que no existe confiscatoriedad.

En todos los casos analizados, la base legal revisada, fue la establecida en el Artículo 41 de la Ley de Régimen Tributario Interno vigente en cada periodo de análisis.

Periodo fiscal 2008: La forma de cálculo para este periodo, consistía en la selección del valor mayor entre: a. El monto del 50\% del impuesto a la renta causado en el periodo fiscal anterior, menos las retenciones que le efectuaron y b. Con datos del periodo anterior, el monto resultado de la sumatoria: $0.2 \%$ del Patrimonio Total, $0.2 \%$ del Total de Costos y Gastos Deducibles, $0.4 \%$ del Activo Total y $0.4 \%$ del Total de Ingresos Gravados, a este valor calculado se resta las retenciones que le efectuaron. La opción b se considera como anticipo mínimo.

En el aspecto del pago, la cancelación se realizaba el 50\% en el mes de Julio del año 2008 y el otro $50 \%$ resultante en el mes de Septiembre del año 2008, teniendo siempre en cuenta que el día se tomaba con relación al noveno dígito del RUC.

Con relación a la devolución: Solamente era susceptible de devolución, a través del pago indebido y en exceso la parte que sobrepase al anticipo mínimo calculado.

Reducción del pago del anticipo mínimo: Existía la posibilidad de solicitar la reducción cuando se llegara a demostrar que las rentas gravadas para el año 2008, serían inferiores a las del año anterior, o en su defecto, cuando se determine que las retenciones efectuadas, superen el impuesto a pagar. Dicha reducción solamente es aplicable sobre el excedente del anticipo mínimo.

Exoneración del pago del anticipo mínimo: Para la exoneración o no pago del anticipo mínimo de Impuesto a la Renta, se debía informar a la Administración Tributaria, que con base en una proyección anual de los resultados de los contribuyentes al 30 de junio, el anticipo mínimo iba a 
ser igual o menor al impuesto causado proyectado.

También se estableció, que las nuevas empresaso sociedades recién constituidas, debían cancelar elanticipo después delsegundo año deoperación efectiva.

Periodo fiscal 2009: En el suplemento del Registro Oficial No. 392 de fecha 30 de julio de 2008, se publicó la Ley Orgánica Reformatoria e Interpretativa a la Ley de Régimen Tributario Interno, al Código Tributario, a la Ley Reformatoria para la Equidad Tributaria del Ecuador y a la Ley de Régimen del Sector Eléctrico, norma en la cual se reformó el artículo 41 de la LORTI.

La forma de cálculo para este periodo, consistía en la selección del valor mayor entre: a. El monto del 50\% del impuesto a la renta causado en el periodo fiscal anterior, menos las retenciones que le efectuaron $y \mathrm{~b}$. Con datos del periodo anterior, el monto resultado de la sumatoria: $0.2 \%$ del Patrimonio Total, $0.2 \%$ del Total de Costos y Gastos Deducibles, $0.4 \%$ del Activo Total y 0.4\% del Total de Ingresos Gravados, a este valor calculado se resta las retenciones que le efectuaron. La opción $\mathrm{b}$ se considera como anticipo mínimo.

En el aspecto del pago, la cancelación se realizaba el 50\% en el mes de Julio del año 2008 y el otro $50 \%$ resultante en el mes de Septiembre del mismo año, teniendo siempre en cuenta que el día se tomaba con relación al noveno dígito del RUC.

Con relación a la devolución: En caso de que al final del ejercicio, no exista impuesto a la renta causado o si este fuera menor a las retenciones más el anticipo pagado, se puede pedir la devolución del saldo a favor, teniendo en cuenta que la parte correspondiente al anticipo mínimo, solo puede devolverse con nota de crédito, y el saldo bajo cualquier mecanismo de devolución.

Exoneración o Reducción del pago del anticipo mínimo: Existía la posibilidad de solicitar la exoneración o reducción del pago de conformidad, con los porcentajes y condiciones establecidos mediante resolución, siempre y cuando se demuestre, que al final del ejercicio se tendría per- didas, o que las rentas gravadas serán inferiores a las del año anterior o que las retenciones en la fuente de renta cubrirán el monto del impuesto a pagar.

También se estableció, que las nuevas empresaso sociedades recién constituidas debían cancelar el anticipo después del segundoaño de operación efectiva.

A continuación se presente un ejemplo de la aplicación normativa en los años 2008 y 2009:

\section{Información del ejercicio fiscal inmediato anterior}

\begin{tabular}{|l|c|}
\hline \multicolumn{1}{|c|}{ Total de Activo } & $\$ 120.000,00$ \\
\hline Total de Patrimonio & $\$ 90.000,00$ \\
\hline Total de Ingresos Gravados & $\$ 30.000,00$ \\
\hline Total de Costos y Gastos Deducibles & $\$ 25.000,00$ \\
\hline Impuesto a la Renta Causado & $\$ 935,00$ \\
\hline Retenciones que le efectuaron & $\$ 300,00$ \\
\hline \multicolumn{2}{|c|}{ Opción A: } \\
\hline (50\% de 935) - 300 : \$ 167,50 \\
\hline Opción B: Sumatoria \\
\hline$(0.4 \% 120.000,00)$ \\
\hline$(0.4 \% 30.0000,00)$ \\
\hline$(0.2 \% 90.0000,00)$ \\
\hline$(0.2 \% 25.0000,00)$ \\
\hline$(830$ - 300: $\$ 530)$ \\
\hline
\end{tabular}

Fuente: Art. 41 Ley de Régimen Tributario Interno Elaborado por Autores

En el presente caso el anticipo a pagar, ya sea en el año 2008 o 2009, es el Anticipo Mínimo conforme la aplicación de la opción $B$, en este caso el valor asciende a $\$ 530,00$. Este rubro para los años 2008 y 2009 se cancelaba en dos cuotas iguales, la primera en julio por $\$ 265.00$ y la segunda en septiembre por $\$ 265.00$. Ejemplo relacionado a la aplicación del derecho a la devolución en los periodos 2008 y 2009. 
AÑO 2008

Resultados del ejercicio 2008

\begin{tabular}{|l|c|}
\hline $\begin{array}{l}\text { (A) Impuesto a la Renta } \\
\text { Causado }\end{array}$ & $\$ 0,00$ \\
\hline $\begin{array}{l}\text { (B) Anticipo Pagado en } \\
\text { Julio y Septiembre 2008 }\end{array}$ & $\$ 200,00$ \\
\hline $\begin{array}{l}\text { (C) Retenciones que le } \\
\text { efectuaron }\end{array}$ & $\$ 100,00$ \\
\hline (A-B-C) Saldo a Favor & $\$ 300,00$ \\
\hline
\end{tabular}

Fuente: Art. 41 Ley de Régimen Tributario Interno Elaborado por Autores

\begin{tabular}{|l|l|}
\hline $\begin{array}{l}\text { Anticipo según fórmula } \\
\text { a (50\% Impuesto Cau- } \\
\text { sado - Retenciones })\end{array}$ & $\$ 200,00$ \\
\hline Anticipo según fórmula \\
b Sumatoria de coeficien- \\
tes (0.4\% Activo Total + \\
$0.4 \%$ Ingresos Gravados + \\
$0.2 \%$ Patrimonio Total + \\
$0.2 \%$ Costos y Gastos De- \\
ducibles) - Retenciones
\end{tabular}

Fuente: Art. 41 Ley de Régimen Tributario Interno Elaborado por Autores

En este caso el Anticipo cancelado en Julio y Septiembre del 2008 corresponde al calculado según la fórmula $\mathrm{A}, \mathrm{y}$ el anticipo mínimo es el calculado según la fórmula $B$.

En el presente caso los valores sujetos a devolución bajo cualquier mecanismo (acreditación en cuenta o nota de crédito) son el Saldo a favor por retenciones $\$ 100,00$ y el excedente del anticipo mínimo, es decir, $\$ 80,00$ (resultado de considerar 200,00 $-120,00)$, en total la devolución asciende a $\$ 180,00$.

El valor no sujeto a devolución de $\$ 120,00$ podía ser compensado con el impuesto a la renta causado de los siguientes cinco ejercicios fiscales.
AÑ̃ 2009

Resultados del ejercicio 2009

\begin{tabular}{|l|c|}
\hline $\begin{array}{l}\text { (A) Impuesto a la Renta } \\
\text { Causado }\end{array}$ & $\$ 0,00$ \\
\hline $\begin{array}{l}\text { (B) Anticipo Pagado en } \\
\text { Julio y Septiembre 2008 }\end{array}$ & $\$ 200,00$ \\
\hline $\begin{array}{l}\text { (C) Retenciones que le } \\
\text { efectuaron }\end{array}$ & $\$ 100,00$ \\
\hline (A-B-C) Saldo a Favor & $\$ 300,00$ \\
\hline
\end{tabular}

Fuente: Art. 41 Ley de Régimen Tributario Interno Elaborado por Autores

\begin{tabular}{|l|l|}
\hline $\begin{array}{l}\text { Anticipo según fórmula } \\
\text { a (50\% }\end{array}$ & Impuesto Cau- \\
sado $\quad$ Retenciones $)$ & $\$ 200,00$ \\
\hline Anticipo según fórmula & \\
b Sumatoria de coeficien- \\
tes (0.4\% Activo Total + \\
$0.4 \%$ Ingresos Gravados + \\
$0.2 \%$ Patrimonio Total + \\
$0.2 \%$ Costos y Gastos De- \\
ducibles $)$ - Retenciones
\end{tabular}

Fuente: Art. 41 Ley de Régimen Tributario Interno

Elaborado por Autores

En este caso el Anticipo cancelado en Julio y Septiembre del 2009 corresponde al calculado según la fórmula $\mathrm{A}, \mathrm{y}$ el anticipo mínimo es el calculado según la fórmula $B$.

En el presente caso los valores sujetos a devolución bajo cualquier mecanismo (acreditación en cuenta o nota de crédito) son el Saldo a favor por retenciones $\$ 100,00$ y el excedente del anticipo mínimo es decir $\$ 80,00$ (resultado de considerar 200,00 - 120,00), en total la devolución bajo cualquier medio asciende a \$180,00.

El valor de $\$ 120,00$ podía ser devuelto solamente a través de la emisión de una nota de crédito con excepción, la que podía ser negociada en cualquier momento, pero redimible a terceros en el plazo de cinco años contados desde la fecha de presentación de la declaración de la que se estableció que le pago fue realizado de manera excesiva. 


\section{Periodo fiscal 2010 hasta la presente fecha:}

Con la promulgación de la Ley $\mathrm{S} / \mathrm{N}$ publicada en el Suplemento del Registro Oficial 94, el 23 de diciembre de 2009, se dio un nuevo hito en la concepción del cálculo y pago del anticipo mínimo de Impuesto a la Renta, toda vez que en esta norma se considera que dicho pago se convierte en pago mínimo y definitivo en el Impuesto a la Renta.

La forma de cálculo desde el año 2010 quedo determinado de la siguiente manera: Con datos del periodo anterior, el monto resultado de la sumatoria: $0.2 \%$ del Patrimonio Total, $0.2 \%$ del Total de Costos y Gastos Deducibles, $0.4 \%$ del Activo Total y $0.4 \%$ del Total de Ingresos Gravados, esta sumatoria se constituye en el anticipo determinado de Impuesto a la Renta.

El pago se estructura de la siguiente forma: $\mathrm{Al}$ valor del anticipo determinado de Impuesto a la Renta se resta las retenciones en la fuente que le han efectuado, el valor resultante de esta operación se cancela en dos cuotas iguales, en los meses de Julio y Septiembre, con relación al noveno dígito del RUC. El saldo pendiente de pago (que es equivalente al valor de las retenciones) se cancela conjuntamente con la declaración del ejercicio fiscal.

Con relación a la devolución: El anticipo mínimo de Impuesto a la Renta, se convierte en pago definitivo por lo que no es susceptible de devolución, lo que se puede devolver es el saldo a favor originado por retenciones en la fuente que lo ha efectuado. El único mecanismo de devolución establecido, es por un ejercicio económico cada trienio, cuando por caso fortuito o fuerza mayor exista una gravedad en la actividad generadora, para lo cual se debe presentar solicitud y está sujeta a revisión y validación de la Administración Tributaria.

Exoneración o Reducción del pago del anticipo mínimo: No existe la posibilidad de solicitar una exoneración o reducción de este pago de ma- nera individual, el único mecanismo establecido, es para casos excepcionales justificados de sectores o subsectores de la economía, que hayan sufrido una drástica disminución de sus ingresos por causas no previsibles y por lo tanto no controlables por ellos.

También se estableció, que las sociedades recién constituidas, inversiones nuevas y personas naturales obligadas a llevar contabilidad, debían cancelar el anticipo después del quinto año de operación efectiva, entendiendo como tal el inicio del proceso productivo y comercial.

Los contribuyentes que se dediquen a actividades económicas con proyectos productivos agrícolas de agroforestería o de silvicultura de especies forestales, con etapas de crecimiento, que superan el año se exoneran del pago del anticipo, mientras no generen ingresos gravados relacionados a la cosecha.

De igual manera, los contribuyentes que tengan como actividad económica el desarrollo de proyectos de software o tecnología, con etapa de desarrollo mayor a un año, se encuentran exonerados en los periodos fiscales que no generen ingresos.

A continuación se presente un ejemplo de la aplicación normativa desde el año 2010:

\section{Información del año 2009}

\begin{tabular}{|l|c|}
\hline Total de Activo & $\$ 120.000,00$ \\
\hline Total de Patrimonio & $\$ 90.000,00$ \\
\hline Total de Ingresos Gravados & $\$ 30.000,00$ \\
\hline $\begin{array}{l}\text { Total de Costos y Gastos De- } \\
\text { ducibles }\end{array}$ & $\$ 25.000,00$ \\
\hline $\begin{array}{l}\text { Impuesto a la Renta Causa- } \\
\text { do }\end{array}$ & $\$ 935,00$ \\
\hline $\begin{array}{l}\text { Retenciones que le efectua- } \\
\text { ron }\end{array}$ & $\$ 300,00$ \\
\hline
\end{tabular}

Fuente: Art. 41 Ley de Régimen Tributario Interno Elaborado por: Autores 
CÁLCULO DEL ANTICIPO DE IR

$(0.4 \% 120.000,00)$

$(0.4 \% 30.0000,00)$

$(0.2 \% 90.0000,00)$

$(0.2 \% 25.0000,00)$

ANTICIPO DE IR : \$ 830,00

Fuente: Art. 41 Ley de Régimen Tributario Interno

Elaborado por: Autores

El valor de \$ 830,00 se convierte en el Anticipo Mínimo de Impuesto a la Renta del año 2010 a Cancelar, la cancelación se realizará en tres cuotas de la siguiente manera:

Cuotas 1 y 2: (Anticipo calculado - Retenciones que le efectuaron) / 2: $(830-300) / 2: \$ 265,00$

Cuota 3 o saldo a pagar: Es equivalente al valor de las retenciones que le efectuaron: \$300,00

\begin{tabular}{|ll|}
\hline CUOTA 1 & $\$ 265,00$ \\
CUOTA 2 & $\$ 265,00$ \\
CUOTA 3 O SALDO A PA- & $\$ 300,00$ \\
GAR & \\
\cline { 2 - 2 } TOTAL A PAGAR & $\$ 830,00$ \\
\hline
\end{tabular}

Fuente: Art. 41 Ley de Régimen Tributario Interno

Elaborado por: Autores

Las cuotas 1 y 2 se cancelan en Julio y Septiembre de 2010 en función del noveno digito del RUC, mientras que la cuota 3 se cancela con la declaración del Impuesto a la Renta del año 2010, a presentarse el siguiente año.

Posteriormente cuando se liquide el Impuesto a la Renta del ejercicio fiscal 2010, se tomará en consideración el efecto del anticipo mínimo calculado para el año 2010.

Supongamos que al finalizar el ejercicio fiscal 2010, se tuvo como resultado que el propio
Impuesto Causado fue de $\$ 600,00$ y que las retenciones que le efectuaron en el ejercicio fiscal ascendieron a $\$ 250,00$, con estos datos la liquidación se debe desarrollar de la siguiente manera:

El impuesto causado definitivo siempre será el valor mayor entre el propio impuesto causado del ejercicio fiscal y el anticipo calculado para el ejercicio fiscal, en el presente caso se tiene:

\begin{tabular}{|l|c|}
\hline $\begin{array}{l}\text { (A) Impuesto Causado del propio ejercicio } \\
\text { 2010: }\end{array}$ & $\$ 600,00$ \\
\hline $\begin{array}{l}\text { (B) Anticipo Calculado para el ejercicio } \\
\text { fiscal 2010: }\end{array}$ & $\$ 830,00$ \\
\hline $\begin{array}{l}\text { (C) Impuesto Causado definitivo el mayor } \\
\text { entre A y B: }\end{array}$ & $\$ 830,00$ \\
\hline
\end{tabular}

Fuente: Art. 41 Ley de Régimen Tributario Interno Elaborado por: Autores

Una vez obtenido este resultado se procede a liquidar el impuesto considerando los créditos tributarios que se pueden compensar, en el presente caso el valor cancelado por las dos cuotas de anticipo en julio de 2010 y septiembre de 2010 por un monto de $\$ 265,00$ cada una, en total de $\$ 530.00$, así como también el crédito tributario por retenciones en la fuente que le efectuaron por un monto de $\$ 250.00$.

\begin{tabular}{|lc|}
\hline (C) IMPUESTO CAUSADO & 830,00 \\
DEFINITIVO & \\
(D) CUOTAS PAGADAS DEL & 530,00 \\
ANTICIPO 2010 & \\
(E) RETENCIONES QUE LE & 250,00 \\
EFECTUARON & \\
(C - D - E) IMPUESTO A PA- & 50,00 \\
GAR & \\
\hline
\end{tabular}

Fuente: Art. 41 Ley de Régimen Tributario Interno Elaborado por: Autores

El resultado del ejercicio fiscal 2010 es un Impuesto a la Renta a pagar de \$50,00, sin embargo lo interesante de esta apreciación, es que se puede validar didácticamente que el Anticipo Mínimo 
siempre se convertirá en el valor mínimo que se deba cancelar por concepto de Impuesto a la Renta.

Este rubro no es objeto de devolución, ya que, como se ha demostrado en ninguna circunstancia y, siempre y cuando, se haya realizado la determinación del Impuesto a la Renta de manera correcta, se pudiera obtener un saldo a favor por concepto del Anticipo de Impuesto a la Renta.

Lo que prevé la norma es una posible devolución cada trienio pero siempre por causa fortuita o fuera mayor de la actividad generadora.

Cambios específicos a partir del año 2010: A partir del año 2010, se han dado ciertas reformas específicas que no han cambiado el sentido y concepción de Impuesto Mínimo del Anticipo de Renta; sin embargo, es importante que se las detalle para que se valide su vigencia en el periodo correcto.

Periodo 2012: En atención a lo dispuesto en la Ley s/n publicada en el Suplemento del Registro Oficial 847 el 10 de diciembre de 2012, se tiene:

Las instituciones financieras privadas y compañías emisoras de tarjetas de crédito, pagarán el 3\% de los ingresos gravables del ejercicio anterior, pudiendo reducirse mediante decreto ejecutivo hasta un $1 \%$.

Periodo 2014: En atención a la disposición reformatoria vigésima, numeral 4 del Código Orgánico Monetario y Financiero, se estableció lo siguiente:

Cualquier contribuyente, que obtenga ingresos bajo la modalidad de comisiones o similares, por la comercialización o distribución de bienes y servicios, únicamente para efectos del cálculo del anticipo en dicha actividad considerarán como ingreso gravable las comisiones percibidas y como costos y gastos deducibles, aquellos distintos a los de los bienes o servicios ofertados.
La Ley Orgánica de incentivos a la producción y prevención del fraude fiscal publicada en el primer suplemento del Registro Oficial 405 de fecha 29 de diciembre de 2014, definió:

Para efectos del cálculo del anticipo de impuesto a la renta, los contribuyentes que tengan activos revaluados, no considerarán para efectuarlo el valor del revalúo efectuado, ni en el activo ni en el patrimonio.

Periodo 2016: La Ley Orgánica para el Equilibrio de las Finanzas Públicas publicada en el suplemento 744 el viernes 29 de abril de 2016 estableció:

Para el cálculodelanticipode Impuestoala Renta, en el caso de las personas naturales obligadas a llevar contabilidad, deben calcular el anticipo únicamente respecto a los rubros que deben ser considerados en la contabilidad de sus actividades empresariales.

La Ley Orgánica de incentivos tributarios, para varios sectores productivos e interpretativos del artículo 547 del COOTAD publicada en el segundo suplemento del Registro Oficial No. 860 de fecha 12 de octubre de 2016 definió:

Con relación a la devolución, cambia la única forma existente hasta esa fecha que era cada trienio y se dispone, que cuando se haya visto afectada significativamente la actividad económica del contribuyente, en el ejercicio económico y bajo la condición de que supere el impuesto causado, se devolverá la parte que exceda el tipo impositivo efectivo promedio de los contribuyentes en general, establecido por la Administración Tributaria mediante resolución.

En el caso de los operadores de transporte público y comercial no se considerará en el cálculo del anticipo tanto en activos, costos, gastos y patrimonio, el valor de las unidades de transporte y sus acoples. 
Tabla $\mathbf{N}^{\circ} 01$

Aporte porcentual de cada rubro en la recaudación de impuesto a la renta desde el periodo 2008 a octubre 2016

\begin{tabular}{|l|c|c|c|c|c|c|c|c|c|c|}
\hline PERIODOS & 2008 & 2009 & 2010 & 2011 & 2012 & 2013 & 2014 & 2015 & 2016 & Promedio \\
\hline $\begin{array}{l}\text { Retenciones en la } \\
\text { fuente }\end{array}$ & $58 \%$ & $52 \%$ & $64 \%$ & $60 \%$ & $63 \%$ & $61 \%$ & $60 \%$ & $56 \%$ & $64 \%$ & $60 \%$ \\
\hline $\begin{array}{l}\text { A la renta empresas } \\
\text { petroleras y otros } \\
\text { NEP }\end{array}$ & $2 \%$ & $3 \%$ & $0 \%$ & $4 \%$ & $2 \%$ & $2 \%$ & $2 \%$ & $1 \%$ & $0 \%$ & $2 \%$ \\
\hline $\begin{array}{l}\text { Anticipos a la renta } \\
\text { Herencias, Legados y }\end{array}$ & $15 \%$ & $15 \%$ & $12 \%$ & $9 \%$ & $8 \%$ & $9 \%$ & $9 \%$ & $7 \%$ & $9 \%$ & $10 \%$ \\
\hline Donaciones & $3 \%$ & $3 \%$ & $3 \%$ & $3 \%$ & $23 \%$ & $24 \%$ & $24 \%$ & $31 \%$ & $3 \%$ & $13 \%$ \\
\hline Personas Naturales & $23 \%$ & $27 \%$ & $20 \%$ & $24 \%$ & $0 \%$ & $0 \%$ & $0 \%$ & $1 \%$ & $24 \%$ & $13 \%$ \\
\hline Personas Jurídicas & $100 \%$ & $100 \%$ & $100 \%$ & $100 \%$ & $100 \%$ & $100 \%$ & $100 \%$ & $100 \%$ & $100 \%$ & $100 \%$ \\
\hline $\begin{array}{l}\text { Impuesto a la Renta } \\
\text { Global }\end{array}$ & & $0 \%$ & $3 \%$ & $4 \%$ & $4 \%$ & $4 \%$ & $0 \%$ & $2 \%$ \\
\hline
\end{tabular}

Fuente: Art. 41 Ley de Régimen Tributario Interno

Elaborado por: Autores

Figura $\mathbf{N}^{\circ} 01$

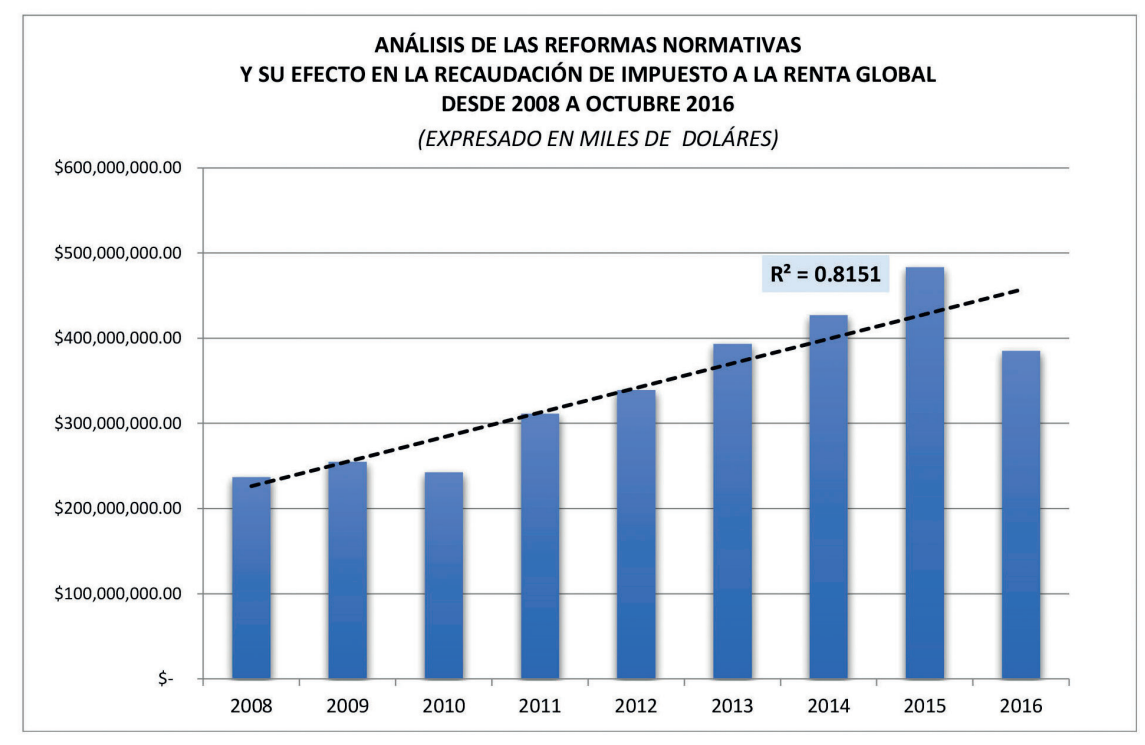

Fuente: Art. 41 Ley de Régimen Tributario Interno

Elaborado por: Autores

Porotra parte se puede apreciar en la Tabla $\mathrm{N}^{\circ} 01$ y Figura $\mathrm{N}^{\circ} 01$ que, el recaudo de impuesto a la renta se ha incrementado en cada periodo, teniendo una tendencia lineal que va en aumento de acuerdo a las metas establecidas por la administración tributaria. 
Tabla $\mathbf{N}^{\circ} 02$

Recaudación de impuesto a la renta por rubro desde el periodo 2008

a octubre 2016

\begin{tabular}{|c|r|r|r|r|r|r|r|}
\hline PERIODOS & $\begin{array}{l}\text { Retenciones } \\
\text { en la fuente }\end{array}$ & $\begin{array}{l}\text { A la renta } \\
\text { empresas } \\
\text { petroleras y } \\
\text { otros NEP }\end{array}$ & $\begin{array}{l}\text { Anticipos a } \\
\text { la renta }\end{array}$ & $\begin{array}{l}\text { Herencias, } \\
\text { Legados y } \\
\text { Donaciones }\end{array}$ & $\begin{array}{l}\text { Personas } \\
\text { Naturales }\end{array}$ & $\begin{array}{l}\text { Personas } \\
\text { Jurídicas }\end{array}$ & $\begin{array}{l}\text { Total Impuesto } \\
\text { la Renta } \\
\text { Global }\end{array}$ \\
\hline 2008 & 1.377 .986 .88 & 35.596 .20 & 52.325 .48 & 3.199 .01 & 60.287 .26 & 39.852 .03 & 2.369 .246 .84 \\
\hline 2009 & 1.332 .928 .91 & 73.394 .21 & 76.192 .41 & 2.798 .71 & 81.632 .59 & 84.798 .13 & 2.551 .744 .96 \\
\hline 2010 & 1.560 .163 .44 & 11.300 .91 & 97.766 .66 & 4.909 .07 & 79.728 .47 & 74.178 .65 & 2.428 .047 .20 \\
\hline 2011 & 1.880 .161 .09 & 124.327 .08 & 267.762 .16 & 2.872 .80 & 92.621 .27 & 744.368 .60 & 3.112 .113 .00 \\
\hline 2012 & 2.140 .087 .19 & 76.599 .50 & 281.762 .73 & 112.530 .89 & 774.230 .45 & 6.026 .13 & 3.391 .236 .89 \\
\hline 2013 & 2.398 .340 .45 & 76.491 .55 & 341.646 .70 & 161.497 .53 & 946.236 .10 & 9.023 .39 & 3.933 .235 .71 \\
\hline 2014 & 2.584 .029 .87 & 76.546 .44 & 380.632 .60 & 177.346 .18 & 1.044 .668 .31 & 10.691 .09 & 4.273 .914 .49 \\
\hline 2015 & 2.702 .012 .10 & 67.982 .92 & 335.432 .47 & 191.933 .77 & 1.506 .833 .46 & 28.917 .71 & 4.833 .112 .43 \\
\hline 2016 & 2.451 .486 .10 & & 347.523 .14 & 7.785 .74 & 134.858 .93 & 911.463 .98 & 3.853 .117 .89 \\
\hline
\end{tabular}

Fuente: Art. 41 Ley de Régimen Tributario Interno

Elaborado por: Autores

Figura $\mathrm{N}^{\circ} 02$

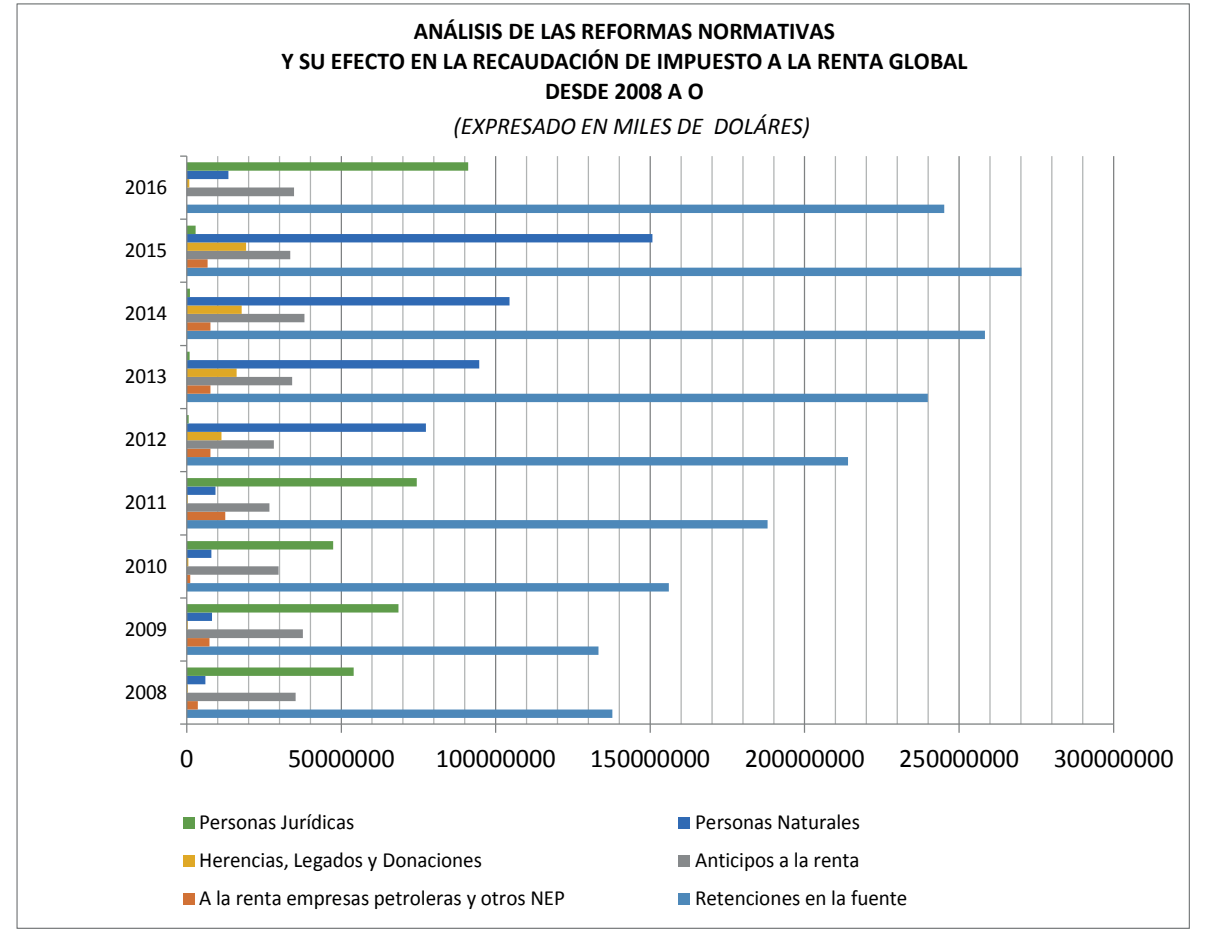

Fuente: Art. 41 Ley de Régimen Tributario Interno

Elaborado por: Autores

Como se puede apreciar en la (Tabla $\mathrm{N}^{\circ} 02$, ver Figura $\mathrm{N}^{\circ}$ 02) los rubros que conforman los recaudos del impuesto a la renta se han incrementado en cada periodo, sin embargo el rubro correspondiente al anticipo propiamente solo representa un promedio del $10 \%$ de la recaudación total, más es importante resaltar que las estadísticas proporcionadas por la Administración Tributaria no consideran el valor generado por el Anticipo del Impuesto a la Renta en la Tercera cuota, ya que el mismo forma parte en las estadísticas del SRI como recaudación del propio Impuesto a la Renta. 


\section{CONCLUSIONES}

1. Durante el año 2008, la norma que regulaba el Anticipo de Impuesto a la Renta ya le daba la connotación de Impuesto Mínimo, otorgando la posibilidad de no pagarlo o incluso a reducir el valor a cancelar. Sin embargo, en el ámbito de devolución, solo se consideraba aplicable sobre el excedente del Anticipo Mínimo.

2. En el año 2009, la norma que regulaba el Anticipo de Impuesto a la Renta ya le daba la connotación de Impuesto Mínimo, otorgando la posibilidad de acceder a una exoneración o reducción del pago, conforme lo dispuesto en la Resolución del SRI. Adicionalmente dicho rubro era objeto de devolución a través de una nota de crédito.
3. Desde el año 2010, hasta la presente fecha la norma legal tributaria le da la connotación de liquidación de un Impuesto Mínimo, estableciéndose desde aquí, que el valor determinado como anticipo de impuesto a la renta se debe cancelar en tres cuotas obligatoriamente, de igual forma no se configura forma alguna de reducción o exoneración del pago del anticipo, mientras que en la devolución se restringe la oportunidad a aplicarlo cada trienio y solo por casos fortuitos o fuerza mayor.

4. En el año 2016, se cambian las reglas para la devolución, eliminándose la modalidad de devolución cada trienio, incorporando una nueva posibilidad, considerando que se haya visto afectada significativamente la actividad económica del contribuyente, en el ejercicio económico y, bajo la condición de que supere el impuesto causado, se devolverá la parte que exceda al tipo impositivo efectivo promedio de los contribuyentes en general, establecido por la Administración Tributaria mediante resolución. 


\section{BIBLIOGRAFIA}

1. CASARES, E. R., GARCIA, S., GUADALUPE, M., RUIZ, G., A., L., \& SOBARZO, H. (2015). Distribución Del Ingreso, Impuestos y Transferencias en México. Un análisis de equilibrio general aplicado. El Trimestre Económico, 523-558.

2. CARBAJO, D. (2013). La tributación de las rentas de capital en América Latina. Panama: CIAT, GIZ, ITC.

3. CASTRO, L., AGUIAR, V., \& SÁENZ, M. (Diciembre de 2013). Análisis de la reforma tributaria en el Ecuador, 2001-2012. SERIE MACROECONOMIA DEL DESARROLLO DE LA CEPAL(143), 2-77.

4. Código Orgánico Monetario y Financiero, Registro Oficial Suplemento, 332 (12 de Septiembre de 2014).

5. Constitución de la República del Ecuador, Registro Oficial 449 (20 de Octubre de 2008).

6. DOMINGUES, J. M., \& CHECA GONZÁlEZ, C. (2013).

7. ConceptodeTributo:UnaPerspectivacomparadaBrasil,España.RevistaDIREITOGV,573-614.

8. GUTIÉRREZ, N. (2015). Impacto de la aplicación del anticipo de Impuesto a la Renta. REVISTA DE INVESTIGACIÓN CONTABLE Quipu Kamayoc (Vol. 23 44), 19.

9. Ley de Régimen Tributario Interno codificado, Registro Oficial Suplemento, 463 (17 de Noviembre de 2004).

10. LeyOrgánica deIncentivosalaProducciónyPrevencióndelFraudeFiscal,RegistroOficialPrimer Suplemento, 405 (29 de Diciembre de 2014).

11. Ley Orgánica de Incentivos tributarios para varios sectores productivos e interpretativos del artículo547 delCOOTAD, RegistroOficialSegundo Suplemento, 860 (12 de Octubre de 2016).
12. Ley Orgánica Para el Equilibrio de las Finanzas Públicas, Registro Oficial Suplemento, 744 (29 de Abril de 2014).

13. Ley Orgánica Reformatoria e Interpretativa a la Ley de Régimen Tributario Interno, al Código Tributario, a la Ley Reformatoria para la Equidad Tributaria del Ecuador y a la Ley de Régimen del Sector Eléctrico, Registro Oficial Suplemento, 392 (30 de Julio de 2008).

14. Ley Reformatoria Para La Equidad Tributaria En El Ecuador, Registro Oficial Tercer Suplemento, 242 (29 de Diciembre de 2007).

15. Ley $\mathrm{S} / \mathrm{N}$, Registro Oficial Suplemento, 94 (23 de Diciembre de 2009).

16. Ley S/N, Registro Oficial Suplemento, 847 (10 de Diciembre de 2012).

17. MASBERNAT, P. (2014). Justicia y sistema tributario. Una mirada desde la perspectiva inglesa. Díkaion, 135-169.

18. Sentencia sobre constitucionalidad del Anticipo Mínimo, 006-13-SIN-CC (Corte Constitucional 17 de Mayo de 2013).

19. PINTO BERNAL, J. J. (2012). Los orígenes del Impuesto Directo y Progresivo en América Latina. Historia y Sociedad, 53-77.

20. RODRÍGUEZ,E.,\&VARGAS,W.(2015).Estrategias de control fiscal para la recaudación tributaria de un órgano municipal. Revista Científica Electrónica de Ciencias Gerenciales / Scientific e-journal of Management Sciences, 3-23. 